\title{
Inability to fully extend the injured elbow: an indicator of significant injury
}

\author{
C. R. E. HAWKSWORTH \& P. FREELAND \\ Accident \& Emergency Department, St John's Hospital at Howden, West Lothian
}

\section{SUMMARY}

Elbow injuries are commonly seen in accident departments. Patients with possible bony injuries to the elbow were assessed to see if inability to fully extend the elbow was a good indicator of bony injury and hence the need for X-ray.

The sensitivity of this test was $90 \cdot 7 \%$ (95\% confidence limits $80.7-100 \cdot 7)$ and the specificity $69.5 \%$ (95\% confidence limits $60 \cdot 3-78 \cdot 7)$. Use of this simple test would reduce both radiography costs and unnecessary exposure to radiation.

\section{INTRODUCTION}

The elbow is a complex joint with hinge movement occurring in a saggital plane at the humero-ulnar/humero-radial articulation and rotation in an axial plane at the humero-radial articulation.

Large numbers of patients attend A\&E with elbow injuries and the authors had previously noted that patients with significant injury usually exhibited loss of full extension of the hinge joint.

This study was designed to assess whether this loss of full extension could be used as a clinical indicator of significant injury. This would be a simple test that could be used by triage nurses or doctors in deciding whom to X-ray.

\section{MATERIALS AND METHODS}

Over a 12-week period all patients presenting to St John's Hospital at Howden with an injury to the elbow were included in the study. A proforma was completed noting the patient's age, sex and mechanism of injury. They were then examined $5 Q E$ 
by a doctor, while standing and with the forearm in a fully supinated position to eliminate any confusion that might be caused by the normal 'carrying angle'. No attempt was made to record the actual range of movement, just whether or not full extension in this position could be achieved.

Radiographs were obtained and reported by a Consultant Radiologist who was unaware of the clinical findings. A significant injury was defined as the presence of a fracture, a joint effusion, or both. This was correlated with the patient's previously noted ability to fully extend the injured elbow.

\section{RESULTS}

During the period of the study, 145 patients attended the A\&E department with elbow injuries representing $2 \cdot 1 \%$ of all new attendances. Forty-five had obvious soft tissue injuries and were not X-rayed. Radiographs were obtained in 100 cases of possible bony injury.

The age and sex were not recorded in 5 patients. Full details were obtained for 95 patients of whom 48 were male and 47 were female. Significant injuries were noted in 54 patients (see Table 1). There was no significant difference in the incidence of bony injury between the sexes $\left(X^{2}=0.546, P>0.9,1\right.$ degree of freedom).

The significant injuries consisted of 6 isolated effusions, 5 isolated fractures and 43 fractures with effusions. The fractures ranged in severity from undisplaced radial head fractures to fracture dislocations at the elbow.

The average age of the patients was 21.5 years with a range from $1-85$ years. Table 2 shows the distribution of patient's ages and the proportion with significant injury."

Just over two-thirds of patients presented with a history of direct trauma to the elbow as opposed to indirect trauma such as a fall on the outstretched hand (see Table 3). A total of $84 \cdot 4 \%$ of the latter had a significant injury compared to $39.7 \%$ of those with direct trauma. This difference is statistically significant $\left(X^{2}=8\right.$, $P>0.001,1$ degree of freedom).

Table 4 shows that loss of full extension was found in 19/46 of those with no significant injury and $49 / 54$ of those with a significant injury.

The sensitivity of loss of extension as an indicator of significant injury is $49 / 54=$ $90.7 \%$ (95\% confidence limits 80.7 to $100 \cdot 7$ ), ie if the patient can fully extend the injured elbow, there is only a $9.3 \%$ chance that he has a significant injury. The

Table 1. Patient's sex and significance of injury

\begin{tabular}{lccr}
\hline & No significant injury & Significant injury & Total \\
\hline Male & 22 & 26 & 48 \\
Female & 23 & 24 & 47 \\
Not specified & 1 & 4 & 5 \\
Total & 46 & 54 & 100 \\
\hline
\end{tabular}


Table 2. Patient's age distribution and significance of injury

\begin{tabular}{ccc}
\hline Age group & Number in group & Number with significant injury (\%) \\
\hline $0-9$ & 28 & $19(67 \cdot 9)$ \\
$10-19$ & 27 & $13(48 \cdot 1)$ \\
$20-29$ & 15 & $3(20 \cdot 0)$ \\
$30-39$ & 9 & $3(33 \cdot 3)$ \\
$40-49$ & 7 & $3(42 \cdot 9)$ \\
$50+$ & 10 & $9(90 \cdot 0)$ \\
\hline
\end{tabular}

Table 3. Loss of full extension and significance of injury

\begin{tabular}{lcc}
\hline & Full extension & Loss of full extension \\
\hline No significant injury & 27 & 19 \\
Significant injury & 5 & 49 \\
Total & 32 & 68 \\
\hline
\end{tabular}

Table 4. Mechanism of injury and significance of injury

\begin{tabular}{lccc}
\hline & No significant injury & Significant injury & Total \\
\hline Direct trauma & 41 & 27 & 68 \\
Indirect trauma & 5 & 27 & 32 \\
\hline
\end{tabular}

specificity of loss of full extension as an indicator of bony injury is $(5+27) / 46=69.5 \%$ (95\% confidence limits 60.3-78.3).

\section{DISCUSSION}

The study was carried out to assess whether loss of extension in patients with injured elbows could be used by Triage nurses in our Department to determine which elbow injuries to X-ray. Provided that care was taken to examine the elbow, with the forearm in the fully supinated position to eliminate any potential confusion caused by the 'carrying angle', this proved a remarkably quick and easy test to perform. By applying this test to the patients in our study only five out of 54 significant injuries would have been 'missed'. These five injuries consisted of two isolated effusions and three undisplaced radial head fractures. Moorewood (1987) suggests that $29 \%$ of traumatic elbow effusions reveal an underlying fracture if re-X-rayed 1 or 2 weeks later, but Quinton et al. (1987) disputes the clinical significance of isolated effusions. In this Department, both isolated effusions and undisplaced radial head fractures are managed in a collar and cuff for 2 weeks and 
then mobilized. At worst, the five 'missed' injuries would have been mobilized $\underset{\Phi}{\overparen{D}}$ 2 weeks earlier, probably with no ill effect.

The study highlighted that other factors may usefully be taken into account $\frac{3}{\mathrm{O}}$ when deciding who to $X$-ray, as age and mechanism of injury appear to have some bearing on the likelihood of significant injury. Those with a history of indirect $\stackrel{\vec{\rho}}{\stackrel{9}{9}}$ trauma were more likely to have a significant injury, as were those over 50 years of $\frac{}{2}$ age. In this latter group, all patients had loss of full extension and would have $\frac{\bar{\sigma}}{\bar{\omega}}$. been X-rayed for that reason. Further clarification of these factors would require $a \frac{\vec{\phi}}{\circ}$ larger study along the lines of that by Dunlop et al. (1986) on ankle inversion injuries.

By applying this simple test, $32 \%$ of patients with elbow injuries, who previously would have been X-rayed, would have been saved unnecessary exposure to radi- $\vec{\omega}$ ation. In this Department, this represents some 200 radiographs per year at a cost $\frac{\Phi}{\Phi}$ of approximately $£ 2000$.

In conclusion, loss of full extension to the injured elbow is a highly sensitive indicator of significant injury and can be used to determine which patients to $\mathrm{X}$-ray with a low risk of missing serious bony injuries.

\section{ACKNOWLEDGEMENTS}

We would like to thank our Medical and Nursing colleagues for their help in the $\vec{\varnothing}$ study and Mrs Linda Campbell for typing the manuscript.

\section{REFERENCES}

Dunlop M. G., Beattie T. F. \& White C. K (1986) Guidelines for selective radiological assessment of inversion ankle injuries. British Medical Journal 293, 603-5.

Morewood, D. J. W (1987) Incidence of unsuspected fractures in traumatic effusions of the elbow joint. British Medical Journal 295, 109-10.

Quinton, D. N., Finlay D. \& Butterworth R. The elbow fat pad sign: a brief report. Journal of Bone and Joint Surgery 69(5), 844-5. 